\title{
Zur Kinetik des monomolekularen Zerfalls organischer Ionen in hohen elektrischen Feldern
}

\author{
H. D. BECKEY und H. KNÖPPEL * \\ Institut für Physikalische Chemie der Universität Bonn \\ (Z. Naturforschg. 21 a, 1920-1930 [1966] ; eingegangen am 27. Juli 1966)
}

\begin{abstract}
The kinetics of unimolecular decomposition of organic ions in the presence of electric fields up to $10^{8} \mathrm{~V} / \mathrm{cm}$ is discussed. The results are deduced from the mass spectra obtained by field ionization of organic molecules. The time interval between formation and decomposition of the ions can be derived from the shape of the mass lines. It is shown that field induced decomposition can occur at a minimum time of about $3 \cdot 10^{-14} \mathrm{sec}$ after field ionization. The optimum time resolution with these experiments, $10^{-12} \mathrm{sec}$, is given by the resolving power of the mass spectrometer.

The results can be interpreted in terms of five different mechanisms:

1. Field induced dissociation. Excitation of the molecular ion to a state above the dissociation limit is possible at high enough fields. Spontaneous dissociation within one vibrational period will then be possible because the excitation energy is contained in the reaction coordinate.

2. Field induced dissociation by tunneling of radicals through the potential barrier. This may occur after excitation of the molecular ion to a state lying in an interval between the dissociation limit and about half a vibrational energy quantum below this, provided the field strength is high enough. The maximum observable life time of ions resulting from this process is about $6 \cdot 10^{-12}$ sec.

3. Field induced dissociation, delayed by re-orientation of the molecular ion. Certain orientations of the molecular ion are favoured with respect to field dissociation. The maximum re-orientation time for a favourable position is of the order $3 \cdot 10^{-12} \mathrm{sec}$.

4. Field induced statistical dissociation. This is due to fluctuation of energy within the ion, as in the case of common reaction kinetics, but with lowering of the dissociation limit by the field. The time intervall for these processes lies between about $10^{-13}$ and $10^{-11}$ sec.

5. Statistical decomposition in the space of low or zero field strength. This process is due to energy fluctuation within the molecular ion excited to a state above the dissociation limit, lowered only by a minute amount by the weak field. The range of life times is about $10^{-11}$ to $10^{-8}$ sec. Processes of the same type, occurring at zero field strength within about $10^{-8}$ and $10^{-6}$ sec, are called - as usually - "metastable" processes.

The processes described here are derived mainly from the FI mass spectra of paraffins, alcohols, ethers and ketones. Dissociation is hindered, in some cases, by high electric fields.
\end{abstract}

In Feldionen-(kurz: FI-) Massenspektren treten bei Benutzung eines Massenspektrometers NiERscher Bauart Massenlinien unterschiedlicher Form auf. Abb. 1 zeigt je ein Beispiel für die Massenlinie eines Molekülions, eines Bruchstückions und eines metastabilen Bruchstückions.

Die Form der Massenlinien von Molekül- und metastabilen Bruchstückionen unterscheidet sich i. A. nicht von derjenigen in Elektronenstoß-Massenspektren. Die Massenlinien der Molekülionen behalten allerdings nur im Bereich nicht zu hoher Feldstärken, wo Ionisation nur in unmittelbarer Nähe von $d_{\min }{ }^{1}$ erfolgt, ihre scharfe, symmetrische Gestalt. Bei sehr hohen Feldstärken, wie sie bei massenspektrometrischen Untersuchungen üblicherweise jedoch nicht verwendet werden, erscheinen auch die Linien von Molekülionen zu kleinen Massen hin ver-

\footnotetext{
* Jetzt EURATOM, Ispra.

1 Zur Def. von $d_{\min }$ s. z. B. H. D. Beckey, Advan. Mass Spectr. Vol. 2, p. 1, Pergamon Press, London 1963.
}

breitert und nehmen bei extrem hohen Feldstärken die Form von metastabilen Peaks an ${ }^{2,3}$.

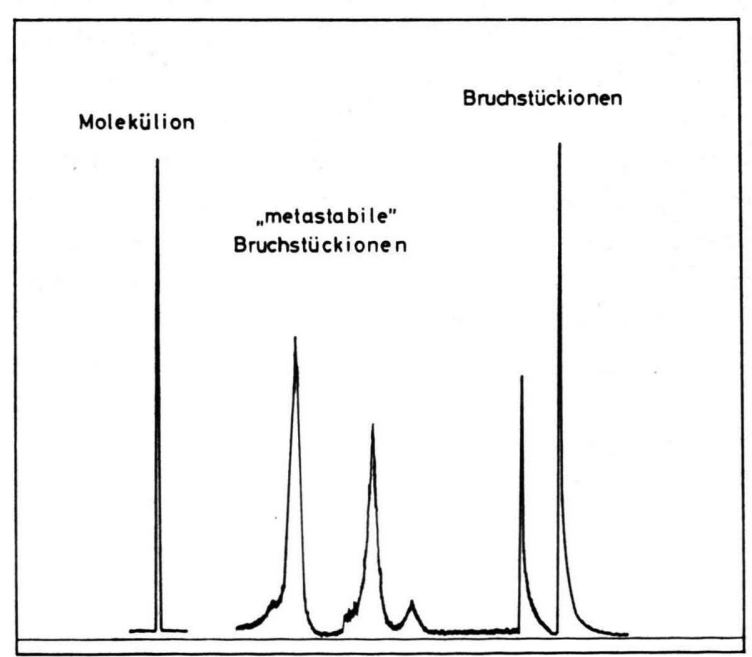

Abb. 1. Typische Form der Ionenstromkurven von Molekülionen, "metastabilen" Bruchstückionen und feldinduzierten Bruchstückionen in FI-Massenspektren. 
Im Gegensatz zu Elektronenstoß-Massenspektren zeigen jedoch die Massenlinien von Bruchstückionenströmen in FI-Spektren in der Regel eine Verbreiterung zu kleinen Massen hin ${ }^{4-6}$. In der vorliegenden Arbeit werden der Zusammenhang zwischen diesen Verbreiterungen und der Lebensdauer der Molekülionen sowie mögliche Ursachen für die Verbreiterung untersucht.

\section{Zusammenhang zwischen der Lebensdauer von Molekülionen und der Form Bruchstück-Ionenstromkurven}

Die Verbreiterung von Bruchstück-Ionenstromkurven wurde zuerst am Bruchstückion der Masse 29 im Massenspektrum des n-Butan gefunden und erklärt ${ }^{4}$. Später wurden theoretische Beziehungen zwischen den Lebensdauern organischer Ionen und den Verbreiterung der Fragmentpeaks abgeleitet. Abb. 2 zeigt schematisch die Äquipotentiallinien zwischen einer auf positivem Potential von $10 \mathrm{kV}$ befindlichen Spitze und einer Kathode. (Die Äquipotentiallinien verdichten sich in Richtung von der Kathode zur Spitze hin wesentlich stärker als in Abb. 2 gezeigt).

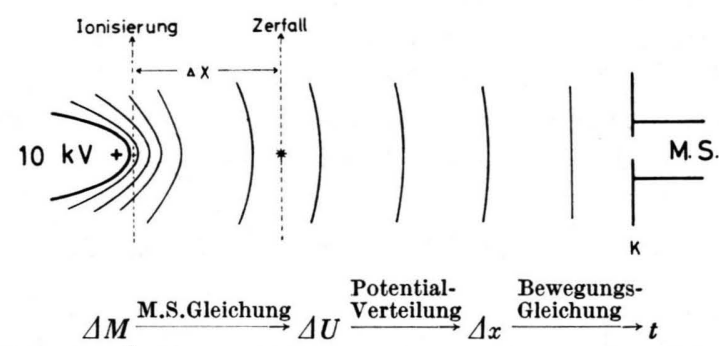

Abb. 2. Äquipotentiallinien zwischen einer auf positivem Potential befindlichen Spitze und einer Kathode (schematisch). $\mathrm{K}=$ Kathode, $\mathrm{MS}=$ Massenspektrometer-Analysatorrohr. Schematische Darstellung der Verknüpfung von $\Delta M$, $\Delta U, \Delta X$ und $t$ (siehe Text).

In unmittelbarer Nähe der Spitze entstehe ein positives Ion durch Feldionisierung eines Moleküls. Das Ion wird durch das elektrische Feld zur Kathode hin beschleunigt und kann in einer gewissen Entfernung von der Spitze dissoziieren. Das elektrische Potential ist am Zerfallsort um einen bestimmten Betrag $\Delta U$ gegenüber dem Wert an der Spitzenoberfläche erniedrigt. Die gebildeten Fragmentionen haben eine entsprechend kleinere Energie als die auf

\footnotetext{
2 R. Gomer, Field Emission and Field Ionisation, Harvard University Press, Cambridge, Mass. 1961, p. 83.

3 J. B

4 H. D. Beckey, Z. Anal. Chem. 170, 359 [1959].
}

der Spitzenoberfläche gebildeten, so daß sie im Massenspektrum bei kleineren Werten auf der Massenskala erscheinen. Aus der Massenspektrometer-Grundgleichung läßt sich eine Beziehung zwischen der beobachteten Massenverschiebung $\triangle M$ und der Potentialdifferenz $\Delta U$ herstellen. Mit Hilfe der Potentialgleichung für eine gegebene Elektrodengeometrie läßt sich die Entfernung $\Delta x$ von der Spitze berechnen, in der der Zerfall des Ions erfolgt. Durch Integration der Bewegungsgleichung des Ions erhält man schließlich eine Korrelation zwischen $\Delta x$ und der Zeit $t$, die zwischen Bildung und Zerfall des Ions verstreicht.

Diese Beziehungen sollen nun im einzelnen abgeleitet werden.

Abb. 3 zeigt schematisch den Potentialverlauf in einer Feldionenquelle, wie sie schon früher beschrieben wurde ${ }^{7}$. Zerfällt ein Molekülion nach einer gewissen Lebensdauer $t$, so bewegt es sich während dieser Zeit im hohen elektrischen Feld vom Ionisierungsort (Spitzenanode) mit dem Potential $U_{0}$ (bezogen auf die Gegenelektrode oder Kathode, s. auch Abb. 3) an einen Ort mit dem Potential $U$. Das dort

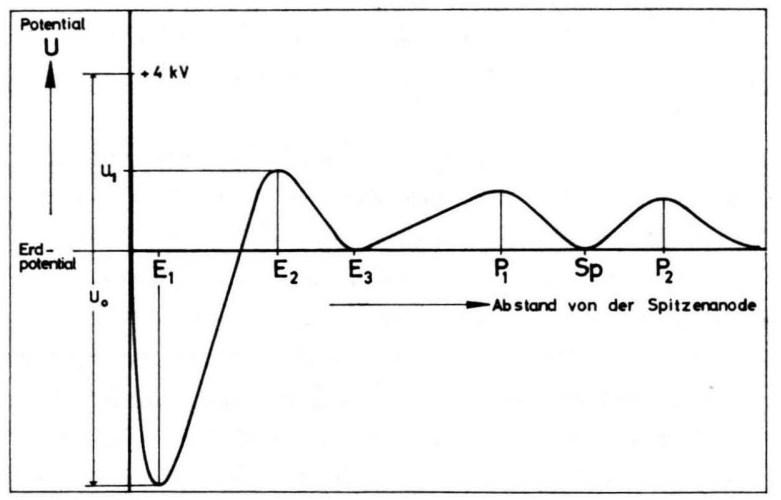

Abb. 3. Potentialverlauf längs der Flugbahn der Ionen in der Feldionenquelle (schematisch). Die Buchstaben an der Abszisse geben die Position der einzelnen Elektroden an. $\mathrm{E}_{1}$ $=$ Kathode $; \mathrm{E}_{2}, \mathrm{E}_{3}=$ Linsenelektroden $; \mathrm{P}_{1}, \mathrm{P}_{2}=$ Ablenkplattenpaare. $\mathrm{S}_{\mathrm{p}}=$ Eintrittsspalt zum Analysator.

entstehende Fragmention erreicht den Analysator des Spektrometers nicht mehr mit der Energie $e\left(U_{0}-U_{\mathrm{g}}\right)$, sondern mit einer geringeren Energie $e U^{*}$, für die gilt :

$$
\begin{aligned}
U^{*} & =\left(U_{0}-U\right) \cdot m / M+U-U_{\mathrm{g}} \\
& =\left(U_{0}-U_{\mathrm{g}}\right)-\left(U_{0}-U\right) \cdot(1-m / M)
\end{aligned}
$$

5 H. D. Beckey, Z. Naturforschg. 16 a, 505 [1961].

${ }^{6}$ H. D. Beckey et al., Advan. Mass Spectr., Vol. 3, Institute of Petroleum, London 1966, p. 35.

7 H. D. Beскеy, Z. Naturforschg. 17 a, 1103 [1962]. 
( $m=$ Masse des Fragmentions, $M=$ Masse des Molekülions). Da die Massenskala mit der Energie $e\left(U_{0}-U_{\mathrm{g}}\right)$ geeicht ist, wird ein Fragmention mit der Energie $e U^{*}<e\left(U_{0}-U_{\mathrm{g}}\right)$ im Massenspektrum nicht bei seiner wahren Masse $m$, sondern bei einer "scheinbaren“ Masse $m^{*}<m$ nachgewiesen, für die gilt :

oder

$$
m^{*}=m U^{*} /\left(U_{0}-U_{\mathrm{g}}\right)
$$

$U^{*}=\left(U_{0}-U_{\mathrm{g}}\right) \cdot m / m$

Setzt man $U^{*}$ aus Gl. (2) in Gl. (1) ein, so erhält man die Potentialdifferenz $\Delta U=U_{0}-U$ zwischen Spitze und Zerfallsort eines Molekülions als Funktion von $m, M, m^{*}, U_{0}$ und $U_{\mathrm{g}}$ :

$$
U_{0}-U=U_{0} B
$$

mit $B=\left(1-m^{*} / m\right)\left(1-U_{\mathrm{g}} / U_{0}\right) /(1-m / M)$.

Da die Lebensdauer $t$ der Molekülionen wiederum über die Gleichung

$$
t=\int_{r_{0}}^{r} \frac{\mathrm{d} s}{v(s)}=\int_{r_{0}}^{r} \frac{\mathrm{d} s}{\sqrt{2 e\left(U_{0}-U(s)\right) / M}}
$$

mit der Potentialdifferenz (3) verknüpft ist, erlaubt die Integration von $\mathrm{Gl}$. (4 a), $t$ in Abhängigkeit von $m, M, m^{*}, U_{0}$ und $U_{\mathrm{g}}$, also insbesondere von $m^{*} \mathrm{zu}$ berechnen.

In Gl. (4 a) ist $r_{0}$ der Abstand des Ionisierungsortes, $r$ der Abstand des Zerfallsortes vom Krümmungsmittelpunkt im Spitzenscheitel, $s$ die Abstandsvariable längs der Flugbahn des Molekülions und $v(s)$ dessen Geschwindigkeit längs dieser Bahn.

BeCKEY ${ }^{5}$ integrierte Gl. (4a), indem er Spitze und Gegenelektrode durch zwei konzentrische Kugeln der Radien $r_{0}$ und $R$ (=Abstand Spitze-Gegenelektrode) ersetzte. Dabei erhält man insbesondere für Werte $m^{*}$, die sehr nahe an $m$ liegen, zu kleine Werte für die Lebensdauer $t$.

Eine völlig analoge Rechnung ergibt genauere Werte für $t$, wenn Spitze und Gegenelektrode durch koaxiale Rotationsparaboloide mit den Scheitelkrümmungsradien $r_{0}$ und $2 R+r_{0}$ angenähert werden und man in Gl. (4 a) für $U(s)$ das Potential auf der beiden Paraboloiden gemeinsamen Symmetrieachse einsetzt.

Dabei ergibt $\operatorname{sich}^{8}$

$$
U_{0}-U(s)=\frac{U_{0}}{\lambda} \cdot \ln \left(2 s / r_{0}-1\right)
$$

\footnotetext{
${ }^{8}$ H. KNöPPEL, Dissertation, Universität Bonn 1965.
}

mit

$$
\lambda=\ln \left(2 R / r_{0}\right) .
$$

Durch die Substitution $x=\left(\ln \left(2 s / r_{0}-1\right)\right)^{1 / 2}$ geht Gl. $(4 \mathrm{a})$ über in

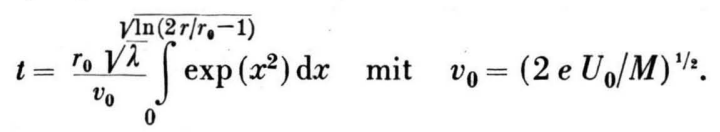

Die obere Grenze in diesem Integral läßt sich mit Hilfe der Gl. (3) und (4 b) durch $\lambda$ und $B$ ausdrücken, so daß man schließlich

$$
t=\frac{r_{0} \sqrt{\lambda}}{v_{0}} \int_{0}^{\sqrt{\lambda B}} \exp \left(x^{2}\right) \mathrm{d} x
$$

erhält. Das Integral in Gl. (4) ist als Funktion der oberen Grenze tabelliert ${ }^{9}$.

Setzt man in Gl. (4) $1-m^{*} / m=1 / A$ ein, wo $A$ das Auflösungsvermögen des verwendeten Spektrometers ist, so erhält man angenähert diejenige Zeit $\Delta t_{0}$, innerhalb derer die zum Peakmaximum beitragenden Bruchstückionen aus den Molekülionen gebildet worden sind. $A$ ist dabei definiert als das Verhältnis der Masse eines Peaks zu dessen Halbwertsbreite, ausgedrückt in Masseneinheiten. Das so definierte Auflösungsvermögen des bei den vorliegenden Untersuchungen verwendeten Spektrometers betrug etwa $A=480$. Damit wird die obere Grenze im Integral in Gl. (4) sehr viel kleiner als eins, so daß innerhalb des Integrationsbereichs $\exp \left(x^{2}\right) \cong 1$ gesetzt werden kann und sich

$$
\Delta t_{0}=\left(\lambda r_{0} / v_{0}\right) \sqrt{\left(1-U_{\mathrm{g}} / U_{0}\right) / A \cdot(1-m / M)}
$$

ergibt.

Für Molekülionen-Massen von etwa 50-150 Masseneinheiten, das angegebene Auflösungsvermögen und Bruchstücke, die gegenüber dem Molekülion wenigstens eine $\mathrm{CH}_{3}$-Gruppe verloren haben, ergeben sich bei den üblicherweise verwendeten Spitzenradien und Spannungen Werte von $\Delta t_{0}$, die etwa zwischen $5 \cdot 10^{-13}$ und $3 \cdot 10^{-12}$ sec liegen. Bei Abspaltung von $\mathrm{H}$ oder $\mathrm{H}_{2}$ ergeben sich größere $\Delta t_{0}$. Werte bis zu etwa $1 \cdot 10^{-11}$ sec.

\section{Zerfallsmechanismen}

Zerfallen Molekülionen nach Zeiten $t>\Delta t_{0}$, so wird die nach Gl. (1) geringere Energie der entstehenden Bruchstückionen vom Spektrometer auf-

9 Siehe z. B. Jahnкe-Emde, Tafeln höherer Funktionen, Verlag Teubner, Leipzig 1948. 
gelöst, und die entsprechenden Massenlinien erscheinen in Richtung kleiner Massen verbreitert. Zerfälle von Molekülionen nach Lebensdauern $t>\Delta t_{0}$ können mehrere Ursachen haben. Sie sollen im folgenden diskutiert und ihr Einfluß, soweit möglich, abgeschätzt werden.

\section{II.1. Einfluß des Tunneleffektes auf die Zerfallszeiten}

Wie Beckey ${ }^{6,10}$ semiklassisch gezeigt und Hiskes ${ }^{11}$ für $\mathrm{H}_{2}{ }^{+}$quantenmechanisch berechnet hat, wird der Potentialverlauf an den Bindungen eines Molekülions vom elektrischen Feld beeinflußt. Die Art und Intensität des Feldeinflusses hängt hauptsächlich von der Orientierung des Molekülions relativ zum elektrischen Feld und von der Verteilung der positiven Ladung im Molekülion ab. Die Potentialkurve einer Bindung wird am stärksten verändert, wenn die positive Ladung an dieser Bindung lokalisiert und das Molekülion in Feldrichtung ausgerichtet ist. Der sich dann ergebende Potentialverlauf ist in Abb. 4 schematisch dargestellt. Der wesentliche Unterschied zum Potentialverlauf ohne Feld liegt darin, daß die Potentialkurve neben einem Minimum auch ein relatives Maximum besitzt. Erfolgt der die Ionisation begleitende FrancK-Condon-Übergang in ein Schwingungsniveau unterhalb dieses Maximums der Potentialkurve, so besteht bei jeder Schwingung eine gewisse Wahrscheinlichkeit $T$ dafür, daß das Molekülion durch quantenmechanischen Tunneleffekt dissoziiert. Auf diese Weise können Molekülionen noch nach Lebensdauern von $t>\Delta t_{0}$ zerfallen. Die kurze Verweilzeit der Ionen im hohen elektrischen Feld bewirkt, daß die Tunnelwahrscheinlichkeit $T$ an einer Bindung einen gewissen unteren Grenzwert überschreiten muß, damit die durch Tunnelzerfall dieser Bindung gebildeten Bruchstückionen einen meßbaren Beitrag zu den verbreiterten Flanken der Ionenstromkurven liefern.

Um den Einfluß des Tunneleffektes auf die Peakverbreiterungen beurteilen zu können, soll versucht werden, diese untere Grenze für die Tunnelwahrscheinlichkeit abzuschätzen und mit ihrer Hilfe den Energiebetrag $H \mathrm{zu}$ ermitteln, um den ein Schwingungsniveau höchstens unter dem Maximum der Potentialkurve liegen darf, damit ein Tunnelzerfall des Molekülions aus diesem Niveau heraus noch als Peakverbreiterung meßbar ist.

10 H. D. BeCKey, Z. Naturforschg. 19 a, 71 [1964].

11 J. R. Hiskes, Phys. Rev. 122, 1207 [1961].

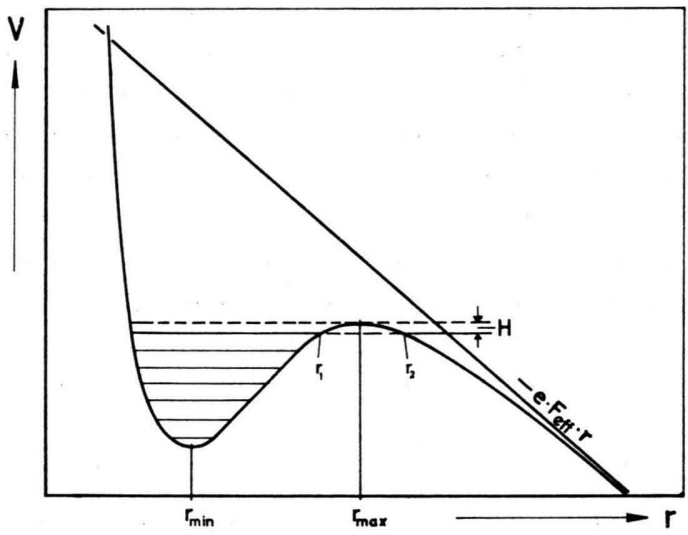

Abb. 4. Potentialverlauf an einer Bindung in einem Molekülion unter dem Einfluß eines hohen elektrischen Feldes (schematisch).

Dazu werde die Felddissoziation einer bestimmten Bindung in einem Molekülion betrachtet, bei der das Bruchstückion der Masse $m$ entsteht. Die auf der Flanke des Fragmentionen-Peaks bei der Masse $m^{*}<m$ nachgewiesenen Bruchstückionen der wahren Masse $m$ entstehen aus Molekülionen der Lebensdauer $t\left(m^{*}\right)$, die nach Gl. (4) berechnet werden kann. Diese Zeit $t$ ist allerdings auf Grund des endlichen Auflösungsvermögens $m^{*} / \Delta m^{*}=A$ des Spektrometers nur bis auf ein kleines Zeitintervall $\Delta t$ bestimmt, so daß zum Ionenstrom bei $m^{*}<m$ Bruchstückionen beitragen können, die innerhalb des Lebensdauer-Intervalls

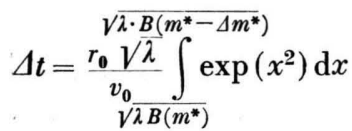

$$
\begin{aligned}
& =\Delta t_{0} \cdot K
\end{aligned}
$$

aus den Molekülionen entstehen. Setzt man

$$
1-m^{*} / m=n / A,
$$

so gilt für den hier interessierenden Bereich von $n=2$ bis ca. $n=10$ näherungsweise

$$
K \simeq \sqrt{n+1}-\sqrt{n} \lesssim 1 / 3 .
$$

Der durch Tunnelzerfall aus einem bestimmten Schwingungsniveau der betrachteten Bindung heraus entstehende Ionenstrom bei der Masse $m^{*}$ beträgt dann

$$
i=q(\dot{n} / p) v \cdot \frac{1}{3} \Delta t_{0} T,
$$

wo $q$ die Elementarladung, $\dot{n}$ die Zahl der pro Zeiteinheit entstehenden Molekülionen, $1 / p$ der Bruchteil 
der davon nach der Lebensdauer $t\left(m^{*}\right)$ noch undissoziierten und das betrachtete Schwingungsniveau besetzenden Molekülionen, $v$ die Schwingungsfrequenz an der betrachteten Bindung und $T$ die Tunnelwahrscheinlichkeit bei Dissoziation aus dem betrachteten Niveau ist.

Da im Massenspektrum nur ein gewisser Bruchteil $1 / s$ der am Auffänger des Spektrometers gemessenen Ionenstromsumme $\Sigma i=q \cdot \dot{n}$ nachgewiesen werden kann, muß für die Tunnelwahrscheinlichkeit $T$

$$
\frac{1}{p} T v \frac{\Delta t_{0}}{3} \geqq \frac{1}{s} \text { bzw. } T \geqq \frac{p}{s} \frac{3}{v \cdot \Delta t_{0}}
$$

gelten, damit der bei $m^{*}$ erscheinende, durch Tunnelzerfall aus dem betrachteten Niveau entstehende Fragmentionenstrom noch nachweisbar ist. Für $v$ gilt $v \leqq 10^{14} \mathrm{sec}^{-1}$ und unter den in der FI-Massenspektrometrie mit Spitzenanoden üblichen Bedingungen - wie oben erwähnt $-\Delta t_{0} \leqq 3 \cdot 10^{-12}$ sec. Daraus folgt für $T$ die Bedingung:

$$
T \geqq p \cdot 10^{-2} / s \text {. }
$$

Für Werte von $T \geqq 10^{-2}$ leistet der Tunneleffekt zu den Verbreiterungen der Fragmentionen-Peaks ebenfalls keinen Beitrag, da praktisch alle Zerfälle innerhalb $\Delta t_{0}$ erfolgen. $T$ muß also für ein bestimmtes Schwingungsniveau im Intervall

$$
10^{-2} \geqq T \geqq p / s \cdot 10^{-2}
$$

liegen, damit Dissoziation aus diesem Niveau zu einer meßbaren Verbreiterung des entsprechenden Fragmentionen-Peaks führt.

Unter der vereinfachenden Voraussetzung, daß die Molekülreste auf beiden Seiten der dissoziierenden Bindung als starre Massen $M_{\mathrm{a}}$ und $M_{\mathrm{b}}$ angesehen werden können, gibt das WKB-Verfahren ${ }^{12}$ eine Möglichkeit, die Tunnelwahrscheinlichkeit $T$ angenähert zu berechnen:

$$
T \simeq \exp \left[-\frac{2 \sqrt{2 \mu}}{\hbar} \cdot \int_{r_{1}}^{r_{2}} \overline{V V(r)}-E \mathrm{~d} r\right]
$$

mit $\quad \mu=M_{\mathrm{a}} M_{\mathrm{b}} /\left(M_{\mathrm{a}}+M_{\mathrm{b}}\right)$.

$E=$ Energie desjenigen Schwingungsniveaus, aus dem der Tunnelzerfall erfolgt. Setzt man für das Potential $V(r)$ - in grober Näherung - eine additive Überlagerung aus dem Morse-Potential

$$
V(r)=D \cdot\left(1-\exp \left[-a\left(r-r_{\min }\right)\right]\right)^{2}
$$

12 G. Wentzel, Z. Phys. 38, 518 [1926]. - L. Brillouin, C. R. Acad. Sci. Paris 183, 24 [1926]. - H. A. Kramers, Z. Phys. 39, 828 [1926]. mit $a=\left(2 \pi^{2} \mu \omega_{\mathrm{e}}^{2} c^{2} / D\right)^{1 / 2} 13$ und dem Potential des effektiven Feldes $F_{\text {eff }}$ ein, so kann man Lage $r_{\max }$ des relativen Maximums und Krümmungsradius $\varrho$ im Maximum der sich ergebenden Potentialkurve (s. Abb. 3) als Funktion der Parameter $D, a$ und $F_{\text {eff }}$ berechnen. (Unter $F_{\text {eff }}$ wird dabei die Neigung des asymptotischen Potentials für große $r$ verstanden, wie es von Beckey ${ }^{7}$ angegeben wurde.) Mit Hilfe von $r_{\max }$ und $\varrho$ läßt $\operatorname{sich} \int_{r_{1}}^{r_{2}} \sqrt{V(r)-E} \mathrm{~d} r$ durch einen übersichtlichen Ausdruck ersetzen, wenn man den Potentialverlauf in der Umgebung des Maximums durch die Parabel

$$
V(r)=V_{\max }-\left(r-r_{\max }\right)^{2} / 2 \varrho
$$

annähert, die im Maximum mit der Potentialkurve zusammenfällt und dort deren Krümmung besitzt. Für ein Schwingungsniveau mit der Energie $E=V_{\max }-H$ (s. Abb. 3) gilt dann:

$$
\int_{r_{1}}^{r_{2}} \sqrt{V(r)-E} \mathrm{~d} r=\pi \cdot H \cdot \sqrt{\varrho / 2}
$$

mit

$\varrho=\left[a^{2} D \alpha(1-\alpha)\right]^{-1}, \alpha=\sqrt{1-2 F_{\text {eff }} / a D}(0<\alpha \leqq 1)$ und folglich

$$
\begin{aligned}
T & =\exp \left(-H \frac{2 \pi}{\hbar} \overline{\sqrt{\varrho \mu}}\right) \\
& ={ }^{10} \exp \left\{-\frac{H}{h c \omega_{\mathrm{e}}} \cdot \frac{4 \pi}{2,3}[2 \alpha(1-\alpha)]^{-1 / 2}\right\}
\end{aligned}
$$

Diese Näherung ist um so besser, je kleiner $H$ ist.

Mit Hilfe von Gl. (9) läßt sich Bedingung (8) in eine entsprechende Bedingung für $H$ umschreiben:

$$
\begin{array}{r}
10^{-2} \geqq{ }^{10} \exp \left\{-\frac{H}{h c \omega_{\mathrm{e}}} \frac{4 \pi}{2,3}[2 \alpha(1-\alpha)]^{-1 / 2}\right\} \\
\geqq \frac{p}{s} \cdot 10^{-2}
\end{array}
$$

oder

$$
\left.2<\frac{H}{h c \omega_{\mathrm{e}}} \cdot \frac{4 \pi}{2,3}-2 \alpha[1-\alpha]\right]^{-1 / 2}<2+\log \frac{s}{p} .
$$

$H \mathrm{mu}$ also in einem Intervall der Größe

$$
\langle H\rangle=h c \omega_{\mathrm{e}} \log (s / p)[2 \alpha(1-\alpha)]^{1 / 2} \cdot \frac{2,3}{4 \pi}
$$

liegen. Der Ausdruck $\alpha \cdot(1-\alpha)$ erreicht für $\alpha=1 / 2$ sein Maximum, so daß die Größe des Intervalls höchstens

$$
\langle H\rangle=0,13 h c \omega_{\mathrm{e}} \cdot \log (s / p)
$$

13 Siehe z. B. G. Herzberg, Spectra of Diatomic Molecules, van Nostrand, Princeton 1950, S. $101 \mathrm{f}$. 
beträgt. Bei den bisher durchgeführten Messungen betrug $s \simeq 10^{4}$. Selbst wenn man $p=1$ setzt, ergibt sich dann, daß $H$ innerhalb eines Energie-Intervalls von der Größe etwa des halben Grundschwingungsquants der betrachteten Bindung liegen muß. Wie Hiskes ${ }^{11}$ zeigen konnte, ändert sich der Abstand der Schwingungsniveaus des $\mathrm{H}_{2}^{+}$-Ions bei Verbiegung der Potentialkurve durch das elektrische Feld nur wenig, und zwar auch nicht in der Nähe des Maximums der vom Feld gestörten Potentialkurve. Nimmt man an, daß sich dieses Ergebnis auch auf Bindungen in größeren Molekülionen übertragen läßt, so liegt innerhalb eines Energie-Intervalls von der durch (10) gegebenen Breite im wesentlichen nur ein Schwingungsniveau. Nur Zerfälle aus diesem Niveau können zu einer (allein durch Tunneleffekt hervorgerufenen) Peakverbreiterung führen. Trotz der sehr groben Näherung dürfte dieses Ergebnis im wesentlichen zutreffen, da in die Abschätzung von $\langle H\rangle$ nur Größenordnungen eingehen.

Ändert sich die Besetzung der Schwingungsniveaus an einer felddissoziierenden Bindung nicht mit der Zeit, so können Zerfälle durch Tunneleffekt allein nur solange geschehen, bis sich das Molekülion im Gebiet so niedriger Feldstärke befindet, daß das Maximum der Potentialkurve sich um etwa $h c \omega_{\mathrm{e}} / 2$ erhöht hat.

Für die bisherigen Untersuchungen der Felddissoziation ist insbesondere die Dissoziation ionisierter C - C-Bindungen interessant. Über das Grundschwingungsquant dieser Bindungen existieren bisher keine Angaben. Das Grundschwingungsquant einer neutralen $\mathrm{C}-\mathrm{C}$-Bindung beträgt etwa $0,1 \mathrm{eV}$. Die Trennungsenergie einer neutralen C - C-Bindung ist mit 3,5-4 eV deutlich größer als die bisher bestimmten Werte für die Trennungsenergie ionisierter $\mathrm{C}$-C-Bindungen, die etwa im Bereich $\lesssim 1 \mathrm{eV}$ liegen ${ }^{14}$. Es scheint deshalb vernünftig, für das Grundschwingungsquant ionisierter C - C-Bindungen einen kleineren Wert anzunehmen als für das der neutralen Bindungen. Schätzt man die obere Grenze von $h c \omega_{\mathrm{e}}$ auf $0,06 \mathrm{eV}$, so wird $h c \omega_{\mathrm{e}} / 2 \lesssim 0,03 \mathrm{eV}$.

Aus der Überlagerung eines geeigneten MorsePotentials mit dem Potential der üblicherweise verwendeten effektiven Feldstärken von $0,2-0,7 \mathrm{~V} / \AA$ läßt sich abschätzen, daß zur Erhöhung des Maximums der Potentialkurve einer ionisierten Bindung

14 F. H. Field u. J. L. Franklin, Electron Impact Phenomena, Academic Press Inc., New York 1957. um den Betrag von $0,03 \mathrm{eV}$ eine Verminderung des effektiven Feldes um maximal $20 \%$ notwendig ist.

In Tab. 1 sind für drei charakteristische Beispiele die Änderungen der Feldstärke längs der Flugbahn der Ionen in Abhängigkeit von der Flugzeit bzw. Lebensdauer angegeben. Daraus folgt, daß die Dissoziation durch Tunneleffekt allein Zerfallszeiten von maximal $6 \cdot 10^{-12} \mathrm{sec}$, d. h. keine Peakverbreiterungen bewirken kann, die größeren Lebensdauern der Molekülionen entsprechen.

\begin{tabular}{|c|c|c|c|}
\hline $\begin{array}{c}\underset{t}{\text { Flugzeit }} \\
\text { sec }\end{array}$ & $\begin{aligned} M & =80, \\
r_{0} & =5000 \AA, \\
U_{0} & =12 \mathrm{kV}\end{aligned}$ & $\begin{array}{c}F / F_{0} \text { für } \\
M=100, \\
r_{0}=8000 \AA \\
U_{0}=16 \mathrm{kV}\end{array}$ & $\begin{aligned} M & =100, \\
r_{0} & =3000 \AA, \\
U_{0} & =6 \mathrm{kV}\end{aligned}$ \\
\hline $1 \cdot 10^{-12}$ & 0,99 & 0,99 & 0,99 \\
\hline $2 \cdot 10^{-12}$ & 0,95 & 0,98 & 0,95 \\
\hline $4 \cdot 10^{-12}$ & 0,83 & 0,92 & 0,83 \\
\hline $6 \cdot 10^{-12}$ & 0,70 & 0,83 & 0,69 \\
\hline $8 \cdot 10^{-12}$ & 0,57 & 0,74 & 0,56 \\
\hline
\end{tabular}

Tab. 1.

Das für FI-Massenspektren organischer Substanzen mit längeren Alkylresten typische Bruchstückion der Masse 29 zeigt in der Regel eine Peakverbreiterung, die Zerfallszeiten $<6 \cdot 10^{-12}$ sec entspricht. Als Ursache für diese Verbreiterung kommt neben dem im folgenden Abschnitt beschriebenen Mechanismus auch der Tunneleffekt in Frage.

\section{II.2. Einfluß der Rotationsbewegung von Molekül- ionen auf die Zerfallszeiten}

Die Dissoziation eines Molekülions im hohen elektrischen Feld hängt wesentlich von seiner Orientierung relativ zum Feld ab, die sowohl die Ladungsverteilung als auch die Verbiegung der Potentialkurven bestimmter Bindungen beeinflußt. Daher kann eine Rotationsbewegung eines Molekülions, die zu einer Änderung seiner Orientierung relativ zum elektrischen Feld führt, eine Änderung der Dissoziationswahrscheinlichkeit bewirken und zu einem späteren Zerfall bzw. einer längeren Lebensdauer des Molekülions führen.

Die Größenordnung der durch diesen Effekt möglichen Verlängerung der Lebensdauer soll am Beispiel der n-Paraffine abgeschätzt werden. Dazu sind in Tab. 2 die klassisch berechneten Zeiten für eine Drehung verschiedener gestreckter Paraffin-Moleküle bei zwei Temperaturen angegeben. Nimmt man an, $\mathrm{da} ß$ die Moleküle in weitgehend gestrecktem Zustand vor der Emissionsspitze rotieren - was auf Grund 
der Untersuchungen von BECKEY ${ }^{7}$ und KNöPPEL und BECKEY ${ }^{15}$ wahrscheinlich ist - so wird ein gewisser Bruchteil der Moleküle im Augenblick einer Orientierung quer zum elektrischen Feld ionisiert werden. Felddissoziation erfolgt dagegen bevorzugt bei Ausrichtung der n-Paraffine längs des Feldes ${ }^{7}$. Der Übergang zwischen beiden Stellungen dürfte etwa die Zeit einer Vierteldrehung in Anspruch nehmen, nach Tab. 2 also Zeiten von $\lesssim 3 \cdot 10^{-12}$ sec.

\begin{tabular}{|l|c|c|c|}
\hline Substanz & $\begin{array}{c}\text { Trägheitsmoment } \\
\text { um eine Achse } \\
\text { durch den Schwer- } \\
\text { punkt des ge- } \\
\text { streckten Moleküls } \\
\perp \text { Kettenrichtung }\end{array}$ & $\begin{array}{c}\text { Dauer einer Drehung } \\
\text { um diese Achse } \\
\text { [sec] }\end{array}$ & $\begin{array}{c}\text { bei } 800^{\circ} \mathrm{K} \\
{[\mathrm{sec}]}\end{array}$ \\
\hline $\mathrm{n}-\mathrm{Hexan}$ & $7,55 \cdot 10^{-38} \mathrm{gcm}^{2}$ & $8,5 \cdot 10^{-12}$ & $5 \cdot 10^{-12}$ \\
\cline { 1 - 1 } $\mathrm{n}-$ Heptan & $1,17 \cdot 10^{-37} \mathrm{gcm}^{2}$ & $1,1 \cdot 10^{-11}$ & $6,5 \cdot 10^{-12}$ \\
\hline n-Oktan & $1,73 \cdot 10^{-37} \mathrm{gcm}^{2}$ & $1,3 \cdot 10^{-11}$ & $8 \cdot 10^{-12}$ \\
\hline
\end{tabular}

Tab. 2.

Bei Molekülen mit verschiedenen endständigen Gruppen können zwischen Ionisation und für Felddissoziation günstiger Ausrichtung Zeiten von der Dauer einer halben Moleküldrehung vergehen, was etwa bei primären Alkoholen möglich erscheint ${ }^{13}$. Molekülionen-Lebensdauern von mehr als etwa $6 \cdot 10^{-12}$ sec und entsprechende Peakverbreiterungen allein auf Grund des beschriebenen Rotationseffektes - ohne gleichzeitige andere Änderungen im Molekülion - sind dagegen unwahrscheinlich.

Die bisher behandelten Ursachen für die Verbreiterungen von Bruchstück-Ionenstromkurven beruhen auf dem Einfluß des hohen elektrischen Feldes auf die Form der Potentialkurven einzelner Molekülbindungen (Tunneleffekt) bzw. deren Dissoziationsenergie (Rotationseffekt), d. h. auf dem „feldinduzierten " Charakter der betrachteten Dissoziationsvorgänge.

\section{II.3. Fluktuation von Anregungsenergie}

Eine weitere Ursache für Molekülionen-Zerfälle nach Lebensdauern $t>\Delta t_{0}$ beruht auf der Fluktuation von Anregungsenergie. Bei Feldionisation wird sie vorzüglich in Form von Schwingungsanregung bei dem die Ionisation begleitenden Franck-CondonÜbergang oder - unter entsprechenden experimen-

15 H. Knöpfel U. H. D. Beckey, Z. Naturforschg. 21 a, 1930 [1966]. tellen Bedingungen - thermisch auf die Molekülionen bzw. Moleküle übertragen. Die Lebensdauern der infolge Energiefluktuation und -akkumulation „statistisch“ zerfallenden Molekülionen hängen nach der statistischen Theorie der Massenspektren (STMS) von der Größe der Anregungsenergie, der Zahl der Freiheitsgrade, auf die sie sich verteilt, und der Größe der Trennungsenergie der zu brechenden Bindung ab. Da diese wiederum u. U. vom elektrischen Feld abhängt, können auch "statistische“ Zerfälle „feldinduziert“ erfolgen. Insbesondere bei erhöhten Temperaturen zerfällt auf diese Weise auch der größte Teil der Molekülionen mit Lebensdauern $t \leqq \Delta t_{0}{ }^{13}$.

Wird die Trennungsenergie einer dissoziierenden Bindung vom elektrischen Feld stark erniedrigt, so erfolgen auch die "statistischen" Zerfälle dieser Bindung vorzüglich im hohen elektrischen Feld. Die dabei resultierenden Peakverbreiterungen sind nicht wesentlich größer als die durch Tunneleffekt oder Molekülrotation möglichen.

Neben feldinduzierten statistischen Zerfällen findet man bei Feldionisation - ebenso wie bei Elektronenstoß-Ionisation - Dissoziationsvorgänge, die zu sog. „metastabilen“ Bruchstückpeaks führen. Dabei handelt es sich um außerhalb des elektrischen Feldes erfolgende relativ langsame Zerfälle von Molekülionen mit Lebensdauern von einigen $10^{-8}-10^{-6}$ sec.

In den FI-Massenspektren einer Reihe organischer Substanzen mit Heteroatomen (z. B. Äther, Amine, Ketone) wurden Fragmentionenpeaks mit Verbreiterungen gefunden, die auf eine dritte Gruppe von statistischen Zerfällen zurückzuführen sind. Diese Zerfälle finden zwar $z$. T. noch im hohen elektrischen Feld, d. h. sehr schnell statt, werden aber vom elektrischen Feld gar nicht oder nur schwach beeinflußt.

Die Bruchstücke, deren Ionenstromkurven die erwähnten Verbreiterungen zeigen, entstehen stets durch Spaltung einer dem Heteroatom benachbarten C-C-Bindung, wobei die positive Ladung am Heteroatom verbleibt. Aus der ElektronenstoßMassenspektrometrie ist bekannt ${ }^{16}$, daß dieser Dissoziationsprozeß durch ein Elektronen-Rearrangement im geladenen Bruchstück energetisch begünstigt wird, das etwa wie folgt formuliert werden kann:

$\mathrm{R}-\mathrm{X}^{+}-\mathrm{CH}_{2}-\mathrm{R}^{\prime} \rightarrow \mathrm{R}-\mathrm{X}^{+}=\mathrm{CH}_{2}+\mathrm{R}^{\prime}$

$$
\longleftrightarrow \mathrm{R}-\mathrm{X}-\mathrm{CH}_{2}^{+}+\mathrm{R}^{\prime}
$$

wo $\mathrm{X}$ für $\mathrm{O}, \mathrm{NH}$ usw. steht.

${ }^{16} \mathrm{~K}$. Biemann, Mass Spectrometry, McGraw-Hill, London 1962. 
Bei Äthern und Aminen tritt die Reaktion (11) auch als metastabiler Prozeß auf, während bei Ketonen ein metastabiler Zerfallsprozeß beobachtet wird, bei dem statt eines neutralen Alkylrestes ein neutrales Paraffin abgespalten, d.h. zusätzlich ein H-Atom umgelagert wird.

Abb. 5 zeigt als Beispiel für die Form der Massenlinien der nach Reaktion (11) entstehenden Bruchstückionen den Peak des Bruchstückions der Masse 59 aus dem Massenspektrum des Diäthyläther. Das Verhältnis von Peakfläche zu Peakhöhe war hier von allen untersuchten Beispielen am größten. Gleichzeitig war die relative Intensität der nach Reaktion (11) innerhalb $\Delta t_{0}$ gebildeten Bruchstückionen beim Diäthyläther am geringsten.

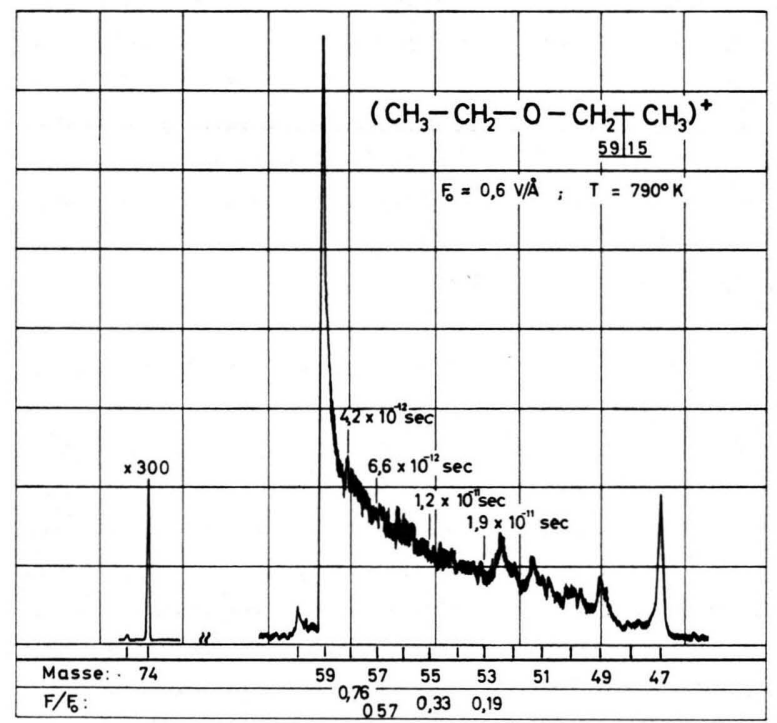

Abb. 5. Ausschnitte aus einem FI-Massenspektrum von Diäthyläther. (Zur weiteren Erläuterung s. Text.)

Die Ionenstromkurve des Bruchstücks der Masse 59 in Abb. 5 erstreckt sich über 12 Masseneinheiten bis zur Masse 47. Bei dieser Masse werden die bei der metastabil erfolgenden Reaktion (11) gebildeten Bruchstückionen registriert. Neben dem Peak der Masse 59 ist zum Vergleich der Linenform der Peak des Molekülions abgebildet.

Mit Hilfe der Beziehung (4) wurde für mehrere Massen $m^{*}$ auf der verbreiterten Flanke des Peaks der Masse 59 die Lebensdauer $t$ der Molekülionen bestimmt, aus denen die Bruchstückionen dieser scheinbaren Massen entstehen. Ebenso wurde das Verhältnis der Feldstärke $F$ am Zerfallsort zur Feldstärke $F_{0}$ am Entstehungsort der Molekülionen mit
Hilfe der Gleichung

$$
F / F_{0}=e^{-\lambda B}
$$

berechnet [wegen der Bedeutung von $\lambda$ und $B$ siehe Gln. (3 a) und (4 b) ]. Für Massen $m^{*}<53$ läßt sich im hier behandelten Beispiel keine eindeutige $\mathrm{Zu}$ ordnung zwischen $m^{*}$ und $t$ sowie $F / F_{0}$ mehr herstellen. Der Grund dafür erhellt aus dem in Abb. 3 gezeigten Potentialverlauf längs der Flugbahn der Ionen:

Diejenigen Bruchstückionen, die an den Schnittpunkten einer Linie $U=$ const mit dem Potentialverlauf entstehen, erscheinen nach Gl. (2) bei derselben Masse $m^{*}$, so daß sich nur für solche Bruchstückionen eine eindeutige Zuordnung zwischen $m^{*}$ und dem Zerfallsort herstellen läßt, die bei Potentialen $>U_{1}$ (s. Abb. 3) gebildet werden. Mit Hilfe von Gl. (2) ergibt sich weiter, daß die Maxima bei den Massen $m^{*}=52,5,51,3,49$ und 47 Zerfällen beim Potential der Elektroden $\mathrm{E}_{2}, \mathrm{P}_{1}$ und $\mathrm{P}_{2}$ und beim Erdpotential (metastabile Zerfälle) entsprechen. Die Maxima erklären sich durch die längere Verweilzeit, d. h. größere Zerfallswahrscheinlichkeit der Molekülionen bei diesen Potentialen. Die Lebensdauer der bei $E_{2}, P_{1}$ und $P_{2}$ zerfallenden Ionen liegt zwischen $10^{-7}$ und $10^{-6}$ sec. Diese Zerfälle erfolgen ebenfalls „metastabil“, völlig außerhalb des hohen elektrischen Feldes.

Eine genauere Untersuchung der betrachteten Ionenstromkurve im Bereich $58 \geqq m^{*} \geqq 53$ zeigt an, daß auch die Zerfälle von Molekülionen mit Lebensdauern zwischen etwa $4 \cdot 10^{-12} \mathrm{sec}$ und $2 \cdot 10^{-11} \mathrm{sec}$, die $\mathrm{z}$. $\mathrm{T}$. noch im hohen elektrischen Feld erfolgen, statistische, vom elektrischen Feld höchstens schwach beeinflußte - also nicht feldinduzierte - Dissoziationsprozesse sind.

Korrigiert man die in Abb. 5 gezeigte Ionenstromkurve, indem man $i$ in jedem Punkt durch das in Gl. (6) berechnete Zeitintervall $\Delta t$ teilt, innerhalb dessen die bei der zugehörigen scheinbaren Masse $m^{*}$ bzw. Zerfallszeit $t$ nachgewiesenen Bruchstückionen gebildet worden sein müssen, dann ist die so erhaltene Kurve der Zerfallsrate $\dot{z}$ (Zahl der Zerfälle pro Zeiteinheit) zu jeder Zeit proportional. Dieses Verfahren ist im hier gegebenen Beispiel natürlich nur im Bereich $m^{*} \geqq 53$ sinnvoll. Abb. 6 zeigt die aus Abb. 5 mit Hilfe des beschriebenen Verfahrens gewonnene Zerfallsrate (bzw. die zu $\dot{z}$ proportionale Größe $i / \Delta t$ ) in logarithmischem Maßstab in $\mathrm{Ab}$ hängigkeit von der Lebensdauer $t$ der Molekülionen. 
$z$ klingt danach exponentiell mit der Lebensdauer ab, wie man es für einen statistischen Zerfallsprozeß mit einer Zerfallskonstanten erwarten sollte. Aus der Tatsache, daß auch nach sehr viel längeren Zeiten $\left(>10^{-7} \mathrm{sec}\right)$ noch Zerfälle stattfinden, muß man

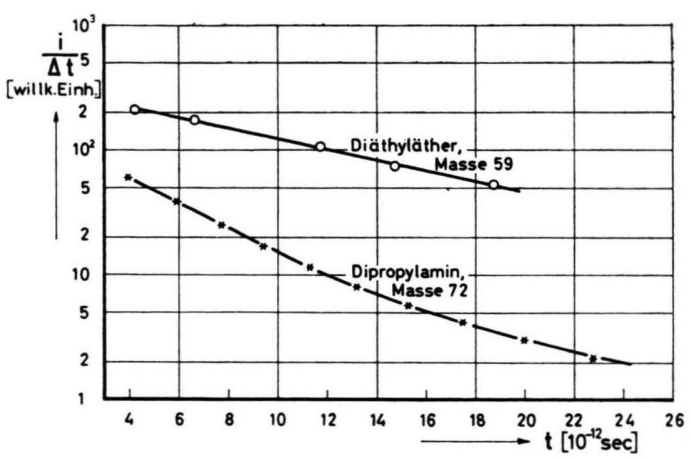

Abb. 6. Zeitliches Abklingen der Zerfallsrate $\dot{z}(\sim i / \Delta t)$ für die Bildung des Bruchstückions der Masse 59 bzw. 72 in einem FI-Massenspektrum vom Diäthyläther bzw. Dipropylamin.

allerdings schließen, daß nicht auf alle Molekülionen dieselbe Energie übertragen wird, sondern die übertragene Energie einer Energieverteilung entspricht. Nach der STMS erzeugt eine solche Energieverteilung ein Spektrum von Zerfallskonstanten $k_{i}$, so daß man ein etwas schwächeres als exponentielles Abklingen der Zerfallsrate $\dot{z}$ mit der Zeit erwarten müßte. Allerdings kann je nach der Form der Energieverteilung die Krümmung der Kurve $\log \dot{z}(t)$ sehr schwach sein. Außerdem muß berücksichtigt werden, daß die Ionenlinse des Massenspektrometers stets so justiert wird, daß die innerhalb $\Delta t_{0}$ entstehenden Bruchstückionen optimal auf den Eintrittsspalt des Massenspektrometers fokussiert werden. Ist die Spitze nicht richtig justiert, so werden bei diesem Verfahren auf Grund der Abbildungsfehler der verwendeten elektrostatischen Linse Bruchstückionen, die später, d.h. in größerer Entfernung von der Spitze entstehen, und deshalb geringere Energie besitzen, schlechter fokussiert, so daß ihre Intensität zu niedrig erscheint.

In der Tat wurde bei Dissoziationsprozessen vom hier behandelten Typ (11) auch schwächeres als exponentielles Abklingen der Zerfallsrate $\dot{z}$ mit der Zeit $t$ beobachtet. Als Beispiel ist in Abb. 6 auch die aus der Ionenstromkurve des Bruchstückions der Masse 72 in einem Massenspektrum des Dipropylamin berechnete Zerfallsrate $\dot{z}$ als Funktion der Lebensdauer $t$ der Molekülionen in logarithmischem Maßstab aufgetragen.
Würde das elektrische Feld wesentlich zur Bildung der betrachteten Fragmentationen beitragen, so müßte man einen stärkeren Abfall der Zerfallsrate $z$ mit $t$ erwarten, wie man ihn etwa beim Bruchstückion der Masse 29 bei Paraffinen beobachtet. Der schwache Einfluß des elektrischen Feldes auf die dissoziierenden Bindungen könnte dadurch bedingt sein, daß die positive Ladung nicht unmittelbar an der dissoziierenden Bindung, sondern zwei Bindungsabstände entfernt am O-Atom bzw. der NH-Gruppe ihre größte Aufenthaltswahrscheinlichkeit besitzt.

\section{II.4. Verzögerung der Molekülionen-Dissoziation durch das elektrische Feld}

Neben feldinduzierten und vom Feld unbeeinflußten Zerfällen sind Dissoziationsprozesse denkbar, die vom elektrischen Feld behindert oder verzögert werden:

a. Die Änderung der positiven Ladungsverteilung in einem Molekülion durch das elektrische Feld, wie sie von Beckey ${ }^{6,10}$ beschrieben wurde, kann die Dissoziationsenergie einer ohne Feld schwachen Bindung erhöhen. Verläßt das Molekülion das hohe elektrische Feld, so stellt sich die ohne Feld vorhandene Ladungsverteilung ein und es erfolgt $u$. U. Dissoziation.

Ein ähnlicher Effekt kann sich ergeben, wenn die positive Ladung an zwei durch eine Bindung verknüpften Radikalen verschieden gut stabilisiert ist, so daß bei Dissoziation dieser Bindung die Dissoziationsenergie davon abhängt, bei welchem Radikal die Ladung verbleibt. In diesem Fall kann bei geeigneter Orientierung des Molekülions bereits eine Polarisation der Ladung zum spitzenfernen Ende der betrachteten Bindung hin zu einer Erhöhung der Lebensdauer des Molekülions gegenüber Dissoziation dieser Bindung führen.

b. Von der Verschiebung bzw. Polarisation der Ladung in einem Molekülion durch das elektrische Feld kann neben der Dissoziationsenergie geeigneter Bindungen eines Molekülions auch die Lage der entsprechenden Potentialkurve bzw. die Größe des Gleichgewichtsabstandes an dieser Bindung geändert werden. Nähert sich dabei der Gleichgewichtsabstand dem derselben Bindung im neutralen Molekül, so wird bei dem die Feldionisation begleitenden FrancKCondon-Übergang die betreffende Bindung u. U. das gesamte Molekülion schwächer schwingungsangeregt als bei Ionisation im feldfreien Raum, z. B. bei Elektronenstoß-Ionisation. 
Auch ohne Änderung der Ladungsverteilung in einem Molekülion kann das elektrische Feld die Potentialkurve einer Bindung zu kleineren Kernabständen hin verschieben, und zwar für den Fall, $\mathrm{da} ß$ sich der Schwerpunkt der positiven Ladung zwischen der Spitzenanode und der betreffenden Bindung befindet ${ }^{6,10}$. Bei der Ionisation im hohen elektrischen Feld wird eine solche Bindung im allgemeinen weniger schwingungsangeregt als bei Ionisation im feldfreien Raum, z. B. durch Elektronenstoß.

Da die Änderung des elektrischen Feldes beim Verlassen der Spitzenanode für die Molekülionen sehr langsam verglichen mit der Schwingungsbewegung der Kerne erfolgt, kann der Übergang der vom Feld geänderten Potentialkurve einer Bindung in die Potentialkurve ohne Feld adiabatisch, d.h. ohne weitere Anregung, erfolgen. Die Lebensdauer eines Molekülions bzw. einer in der beschriebenen Weise vom elektrischen Feld beeinflußten Bindung gegenüber einem statistischen Zerfall kann so erheblich verlängert werden.

Ein Zerfall, der wahrscheinlich infolge des unter a. beschriebenen Einflusses der Änderung der positiven Ladungsverteilung durch das elektrische Feld verzögert erfolgt, wurde bei 1-Pentanol beobachtet. Er verursacht im FI-Massenspektrum dieser Substanz einen über einen weiten Massenbereich „verschmierten" Peak, wie Abb. 7 ihn zeigt.

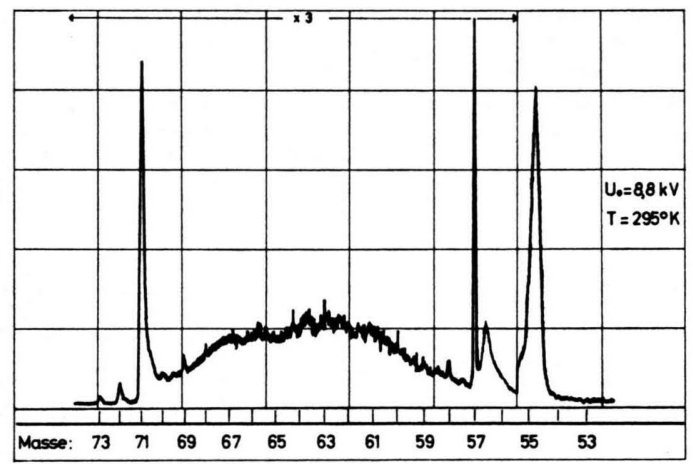

Abb. 7. Ausschnitt aus einem FI-Massenspektrum von 1-Pentanol.

Die Lage des breiten Maximums in Abb. 7, das offensichtlich mit dem Bruchstückionenpeak der Masse 71 zusammenhängt, sowie der Umstand, daß Ionen der Masse 71 metastabil durch die Reaktion

$$
89^{+} \rightarrow 71^{+}+18
$$

gebildet und bei Masse 56,6 nachgewiesen werden, lassen vermuten, daß auch dieses Maximum infolge Reaktion (13) durch Ionen der Masse 71 entsteht.

Für die Ionen der Masse 89 muß die Struktur

$$
\mathrm{H}_{3} \mathrm{C}-\mathrm{CH}_{2}-\mathrm{CH}_{2}-\mathrm{CH}_{2}-\mathrm{CH}_{2}-{ }^{+} \mathrm{O}\left\langle\frac{\mathrm{H}}{\mathrm{H}}\right.
$$

angenommen werden. Es befinde sich die Ladung, wie angedeutet, an der $\mathrm{OH}_{2}$-Gruppe, wobei das Ion unter dem Einfluß der im hohen elektrischen Feld an ihr angreifenden Сочцомв-Kraft so gestreckt wird, daß sich die geladene Gruppe jeweils am spitzenfernen Ende des Ions befindet. Gelangt das Ion in Gebiete genügend kleiner Feldstärke, so kann die Ladung möglicherweise zum Alkylrest übertreten, der eine kleinere Ionisierungsenergie besitzt als die $-\mathrm{OH}_{2}$-Gruppe. Danach kann - evtl. unter Mitwirkung von Fluktuation von Schwingungsenergie Dissoziation erfolgen, wobei Wasser abgespalten wird.

Berechnet man aus der in Abb. 7 gezeigten Ionenstromkurve die Zerfallsrate $\dot{z}$ unter der Annahme, daß die Ionen im wesentlichen nur durch Reaktion (13) entstehen, so erhält man die in Abb. 8 gezeigte Abhängigkeit der Zerfallsrate von der Lebensdauer $t$ der Mutterionen der Masse 89. Auf der Abszisse sind neben $t$ auch $F / F_{0}$ und die scheinbaren Massen $m^{*}$ aufgetragen. Die Zerfallsrate geht bei $m^{*}=70$ durch ein Minimum. Es läßt sich so interpretieren, da $\beta$ bei kleinen $t$ bzw. nahe der Spitzenoberfläche noch andere Prozesse als (13) zur Bildung von Ionen der Masse 71 führen - so etwa die Ionisierung neutraler Radikale - oder daß die Ionen der Masse 89 im Augenblick ihrer Entstehung eine für die Dissoziation nach (13) günstige Orientierung zum Feld besitzen, die dann durch ihre Ausrichtung im Feld gestört wird.

Das Maximum der Zerfallsrate wird bei der Feldstärke $F \simeq 0,3 F_{0}$ erreicht. Die Scheitelfeldstärke $F_{0}$ betrug bei der der Abb. 8 zugrunde liegenden Messung etwa $0,45 \mathrm{~V} / \AA$. Das breite Maximum in Abb. 7 könnte danach durch den unter a. beschriebenen Mechanismus bedingt sein, wenn die Ladung am Alkylrest um etwa $0,15 \mathrm{eV}$ besser stabilisiert ist als an der $\mathrm{OH}_{2}$-Gruppe. Die exponentielle Abnahme der Zerfallsrate zu großen $t$-Werten hin läßt wieder auf einen statistischen Zerfall schließen.

Ein Beispiel für die unter b. beschriebene dissoziationshemmende Wirkung des elektrischen Feldes 
dürfte das Auftreten eines verhältnismäßig intensiven Molekülionen-Peaks im FI-Spektrum des Neopentan sein ${ }^{6}$. Während bisher in Elektronenstoß-Massenspektren auch unterhalb des Appearence-Potentials der Bruchstückionen der Masse 57, die den intensivsten Peak verursachen, keine Molekülionen nachgewiesen werden konnten, erreichen sie in FI-Spektren relative Intensitäten von einigen Prozent.

Der Deutschen Forschungsgemeinschaft und dem Verband der Chemischen Industrie danken wir für die großzügige Förderung der vorliegenden Arbeit durch Sachbeihilfen.

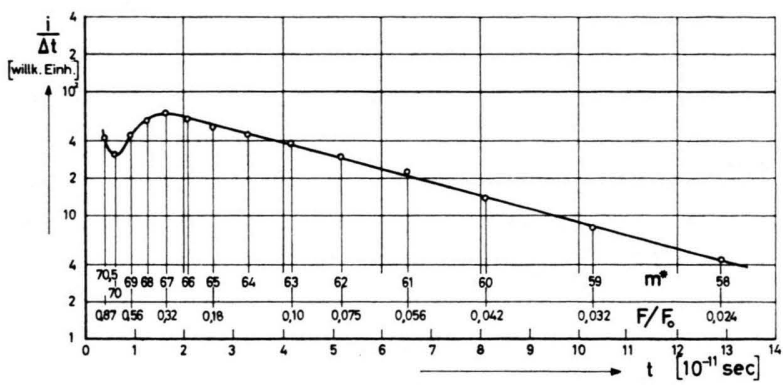

Abb. 8. Abhängigkeit der Zerfallsrate $z(\sim i / \Delta t)$ für die Bildung des Bruchstückions der Masse 71 aus Ionen der Masse 89 von der Lebensdauer dieser Ionen. $i / \Delta t$ wurde aus der in Abb. 7 gezeigten Ionenstromkurve berechnet.

\title{
Zur Temperaturabhängigkeit der Felddissoziation von Molekülionen
}

\author{
H. KNöPPEL * und H. D. BECKEY \\ Institut für Physikalische Chemie der Universität Bonn \\ (Z. Naturforschg. 21 a, 1930-1945 [1966] ; eingegangen am 27. Juli 1966)
}

\begin{abstract}
The temperature dependence of field dissociation of organic molecular ions as described in this paper can be interpreted qualitatively by means of simple models.

Very fast decomposition processes, occurring about $10^{-12} \mathrm{sec}$ after field ionization, are not only due to the lowering of the dissociation energy by the field, but also due to fluctuation of vibrational energy, especially at higher temperatures.

The fragment ion insensities are increased by formation of adsorbed layers on the tip anode at low temperatures, especially with substances having high dipole moments.

In the case of molecules containing a polar group and an alkyl chain, only the former is ordered preferrentially, with the dipole moment parallel to the field lines, whereas the latter is relatively mobile in the electric field.

The relative fragment ion intensities depend on the orientation of the alkyl chain relatively to the polar group, however, this effect does not depend appreciably on temperature because of the mobility of the alkyl chain. The only exception from this rule was observed with pentanol. The pentanol molecules are adsorbed at the tip anode with the alkyl chains preferrentially parallel to each other. This leads to a preferred formation of $\mathrm{C}_{2} \mathrm{H}_{5}{ }^{+}$fragment ions. With increasing temperature, the adsorbed layer is removed and a statistical orientation of the alkyl chain becomes more important, thus reducing the relative $\mathrm{C}_{2} \mathrm{H}_{5}{ }^{+}$abundance.
\end{abstract}

Die Entstehung von Bruchstückionen im Feldionen-(abgekürzt: FI-) Massenspektrum des n-Butans wurde auf Grund einer Analyse der Form der Massenlinien von BECKEy auf einen Felddissoziationseffekt zurückgeführt ${ }^{1}$. Felddissoziation wurde danach bei vielen organischen Ionen gefunden. Die FIMassenspektrometrie erwies sich dabei als eine zur Untersuchung des Dissoziationsverhaltens von Mole-

* Jetzt EURATOM-Ispra.

1 H. D. Beckey, Z. Anal. Chem. 170, 359 [1959].

2 H. D. BeскеY, Z. Naturforschg. 16 a, 505 [1961].

${ }^{3}$ H. D. Beckey, Z. Naturforschg. 17 a, 1103 [1962].

4 H. D. Beckey, Z. Naturforschg. 19 a, 71 [1964]. külionen in hohen elektrischen Feldern geeignete Methode. In einer Reihe von Veröffentlichungen ${ }^{2-7}$ wurde insbesondere die Abhängigkeit der Felddissoziation organischer Molekülionen von der elektrischen Feldstärke untersucht. Diese Untersuchungen ergaben Aufschlüsse über den Einfluß des elektrischen Feldes auf die Verteilung der positiven Ladung in den Molekülionen, deren starken Einfluß

5 H. D. Beckey U. P. Schulze, Z. Naturforschg. 20 a, 1329 [1965].

${ }^{6}$ H. D. Beckey u. P. Schulze, Z. Naturforschg. 20 a, 1335 [1965].

7 H. D. Beckey et al., Advan. Mass Spectr., Vol. 3, Institute of Petroleum, London 1966, p. 35. 